\title{
Large Solutions for Harmonic Maps in Two Dimensions`
}

\section{Haim Brezis ${ }^{1}$ and Jean-Michel Coron ${ }^{2}$}

1 Département de Mathématiques, Université Paris VI, 4, Place Jussieu, F-75230 Paris Cedex 05, France

2 Département de Mathématiques, Ecole Polytechnique, F-91128 Palaiseau Cedex, France

\begin{abstract}
We seek critical points of the functional $E(u)=\int_{\Omega}|\nabla u|^{2}$, where $\Omega$ is the unit disk in $\mathbb{R}^{2}$ and $u: \Omega \rightarrow S^{2}$ satisfies the boundary condition $u=\gamma$ on $\partial \Omega$. We prove that if $\gamma$ is not a constant, then $E$ has a local minimum which is different from the absolute minimum. We discuss in more details the case where $\gamma(x, y)=\left(R x, R y, \sqrt{1-R^{2}}\right)$ and $R<1$.
\end{abstract}

\section{Introduction}

Let $\Omega=\left\{(x, y) \in \mathbb{R}^{2} ; x^{2}+y^{2}<1\right\}$ and $S^{2}=\left\{(x, y, z) \in \mathbb{R}^{2} ; x^{2}+y^{2}+z^{2}=1\right\}$. Let $\gamma: \partial \Omega \rightarrow S^{2}$ be given and assume that $\gamma$ is the restriction to $\partial \Omega$ of some function in $H^{1}\left(\Omega ; S^{2}\right)^{1}$. We set

$$
E(u)=\int_{\Omega}|\nabla u|^{2} \quad \text { for } \quad u \in H^{1}\left(\Omega ; \mathbb{R}^{3}\right)
$$

and

$$
\mathscr{E}=\left\{u \in H^{1}\left(\Omega ; S^{2}\right) ; u=\gamma \quad \text { on } \quad \partial \Omega\right\} .
$$

We seek critical points of $E$ on $\mathscr{E}$. It is obvious that there exists some $\underline{u} \in \mathscr{E}$ such that

$$
E(\underline{u})=\operatorname{Inf}_{\mathscr{\delta}} E .
$$

Our first result is the following:

Theorem 1. If $\gamma$ is not a constant, there exists a critical point of $E$ on $\mathscr{E}$ which is different from $\underline{u}$.

* Work partially supported by US National Science Foundation grant PHY-8116101-A01

1 We use the standard notation for Sobolev spaces:

$H^{1}\left(\Omega ; \mathbb{R}^{3}\right)=\left\{u \in L^{2}\left(\Omega ; \mathbb{R}^{3}\right) ; \quad u_{x}, u_{y} \in L^{2}\left(\Omega ; \mathbb{R}^{3}\right)\right\}$ and

$H^{1}\left(\Omega ; S^{2}\right)=\left\{u \in H^{1}\left(\Omega ; \mathbb{R}^{3}\right) ; \quad u(x, y) \in S^{2}\right.$ a.e. on $\left.\Omega\right\}$ 
In order to prove Theorem 1, we introduce

$$
Q(u)=\frac{1}{4 \pi} \int_{\Omega} u \cdot u_{x} \wedge u_{y}
$$

for $u \in L^{\infty}\left(\Omega ; \mathbb{R}^{3}\right)$ with $u_{x}, u_{y} \in L^{2}\left(\Omega ; \mathbb{R}^{3}\right)$ and we observe (see Lemma 1 ) that

$$
Q\left(u_{1}\right)-Q\left(u_{2}\right) \in \mathbb{Z} \quad \forall u_{1}, u_{2} \in \mathscr{E} .
$$

For every $k \in \mathbb{Z}$ we define the class $\mathscr{E}_{k}=\{u \in \mathscr{E} ; Q(u)-Q(\underline{u})=k\}$. Each class $\mathscr{E}_{k}$ is (non-empty) closed and open in $\mathscr{E}$ for the topology induced by the norm of $H^{1}\left(\Omega ; \mathbb{R}^{3}\right)$.

In order to find other critical points of $E$ on $\mathscr{E}$ it is tempting to consider

$$
\underset{\substack{\mathscr{E}_{k} \\ \text { Inf } E}}{\text { for }} k \neq 0 .
$$

When trying to prove that $\operatorname{Inf} E$ is achieved one encounters a major difficulty due to the fact that $\mathscr{E}_{k}$ is not closed under weak $H^{1}$ convergence. Nevertheless we shall prove that at least one of the two infima $\operatorname{Inf}_{\mathscr{E}_{1}} E$ or $\underset{\mathscr{E}-1}{\operatorname{Inf}} E$ is achieved. The argument involves some ideas used by the authors in [2]; related difficulties also occur in [1,3, $7,8,11]$. Notice that the assumption " $\gamma$ is not a constant" in Theorem 1 is essential. Indeed when $\gamma=C$ is a constant, Lemaire [6] has proved that $u \equiv C$ is the only critical point of $E$ on $\mathscr{E}$.

For simplicity we consider only maps with values into $S^{2}$. The same result holds if $S^{2}$ is replaced by a Riemannian surface homeomorphic to $S^{2}$ (see Remark 2).

The paper is organized as follows: In Sect. 1 we present some technical lemmas. In Sect. 2 we prove Theorem 1. In Sect. 3 we discuss a simple example, namely

$$
\gamma(x, y)=\left(\begin{array}{c}
R x \\
R y \\
\sqrt{1-R^{2}}
\end{array}\right) \text { with } R<1
$$

We prove (see Theorem 2) that $\operatorname{Inf} E$ is not achieved, except when $k=0$ and $k=-1$. We have collected in the Appendix various useful facts and in particular an important density result due to $\mathrm{R}$. Schoen and K. Uhlenbeck [10].

After our work was completed we learned that J. Jost [5] has obtained independently a result similar to our Theorem 1 .

\section{Some Technical Lemmas}

We start with

Lemma 1. Assume $u_{1}, u_{2} \in \mathscr{E}$. then $Q\left(u_{1}\right)-Q\left(u_{2}\right) \in \mathbb{Z}$.

Proof. We consider $w: \mathbb{R}^{2} \rightarrow S^{2}$ defined as follows:

$$
\left\{\begin{array}{l}
w(x, y)=u_{1}(x, y) \text { if } x^{2}+y^{2}<1 \\
w(x, y)=u_{2}\left(\frac{x}{x^{2}+y^{2}}, \frac{y}{x^{2}+y^{2}}\right) \text { if } x^{2}+y^{2}>1
\end{array}\right.
$$


It is easy to check that $w \in L^{\infty}\left(\mathbb{R}^{2} ; S^{2}\right), w_{x}, w_{y} \in L^{2}\left(\mathbb{R}^{2} ; \mathbb{R}^{3}\right)$ and

$$
\frac{1}{4 \pi} \int_{\mathbb{R}^{2}} w \cdot w_{x} \wedge w_{y}=Q\left(u_{1}\right)-Q\left(u_{2}\right)
$$

On the other hand if $\phi \in C^{\infty}\left(\mathbb{R}^{2} ; S^{2}\right)$ and $\phi$ is constant far out then

$$
\frac{1}{4 \pi} \int_{\mathbb{R}^{2}} \phi \cdot \phi_{x} \wedge \phi_{y} \in \mathbb{Z} .
$$

In fact this integer is the degree of the map $\phi \circ \pi: S^{2} \rightarrow S^{2}$, where $\pi: S^{2} \rightarrow \mathbb{R}^{2}$ is a stereographic projection (see for example the analytic expression of the degree given in [9]). It follows by density (see Lemma A.1) that

$$
\frac{1}{4 \pi} \int_{\mathbb{R}^{2}} \phi \cdot \phi_{x} \wedge \phi_{y} \in \mathbb{Z} \quad \forall \phi \in L^{\infty}\left(\mathbb{R}^{2}, S^{2}\right) \text { with } \phi_{x}, \phi_{y} \in L^{2}\left(\mathbb{R}^{2}, \mathbb{R}^{3}\right),
$$

and thus we obtain the conclusion of Lemma 1.

Our next lemma plays a crucial role in the proof of Theorem 1. We assume now that $\gamma$ is not a constant and we fix some $\underline{u} \in \mathscr{E}$ such that $E(\underline{u})=\operatorname{Inf}_{\mathscr{E}} E$.

Lemma 2. There is some $v \in \mathscr{E}$ such that

$$
|Q(v)-Q(\underline{u})|=1
$$

and

$$
E(v)<E(\underline{u})+8 \pi .
$$

Proof. By Morrey's regularity theory we know that $\underline{u} \in C^{\infty}\left(\Omega ; \mathbb{R}^{3}\right)$. Since $\gamma$ is not a constant it follows that $\nabla \underline{u}\left(x_{0}, y_{0}\right) \neq 0$ for some point $\left(x_{0}, y_{0}\right) \in \Omega$. Rotating coordinates in the $(x, y)$ plane we may always assume that

$$
\underline{u}_{x}\left(x_{0}, y_{0}\right) \cdot \underline{u}_{y}\left(x_{0}, y_{0}\right)=0 .
$$

[Indeed, if we set

$$
x^{\prime}=(\cos \theta) x+(\sin \theta) y, \quad y^{\prime}=(-\sin \theta) x+(\cos \theta) y,
$$

we find

$$
\left.u_{x^{\prime}} \cdot u_{y^{\prime}}=-\left(\left|u_{x}\right|^{2}-\left|u_{y}\right|^{2}\right) \sin \theta \cos \theta+u_{x} u_{y}\left(\cos ^{2} \theta-\sin ^{2} \theta\right)\right] .
$$

In addition we have

$$
\underline{u} \cdot \underline{u}_{x}=\underline{u}^{\prime} \cdot \underline{u}_{y}=0 \text { on } \Omega,
$$

since $|\underline{u}|^{2}=1$ on $\Omega$.

Therefore we may choose an orthonormal basis $(\mathbf{i}, \mathbf{j}, \mathbf{k})$ in $\mathbb{R}^{3}$ such that (in the basis $(\mathbf{i}, \mathbf{j}, \mathbf{k}))$

$$
\underline{u}\left(x_{0}, y_{0}\right)=\left(\begin{array}{l}
0 \\
0 \\
1
\end{array}\right), \quad \underline{u}_{x}\left(x_{0}, y_{0}\right)=\left(\begin{array}{l}
a \\
0 \\
0
\end{array}\right), \quad \underline{u}_{y}\left(x_{0}, y_{0}\right)=\left(\begin{array}{l}
0 \\
b \\
0
\end{array}\right)
$$

with $a \geqq 0, b \geqq 0$ and $a+b \neq 0$. (Notice that the basis $(\mathbf{i}, \mathbf{j}, \mathbf{k})$ could possibly have 
a different orientation than the canonical basis of $\mathbb{R}^{3}$ which was used to define $Q$.) Let $\varepsilon>0$ be small enough. We define a function $u^{\varepsilon}: \Omega \rightarrow \mathbb{R}^{3}$ in the following way:

Let $\left.r=\left[x-x_{0}\right)^{2}+\left(y-y_{0}\right)^{2}\right]^{1 / 2}$ and $\theta$ such that $x-x_{0}=r \cos \theta, y-y_{0}=r \sin \theta$. We $\operatorname{set}^{(1)}$

a) If $r>2 \varepsilon$,

$$
u^{\varepsilon}(x, y)=\underline{u}(x, y) \text {. }
$$

b) If $r<\varepsilon$, we set (in the basis (i, $\mathbf{j}, \mathbf{k})$ )

$$
u^{\varepsilon}(x, y)=\frac{2 \lambda}{\lambda^{2}+r^{2}}\left(\begin{array}{c}
x-x_{0} \\
y-y_{0} \\
-\lambda
\end{array}\right)+\left(\begin{array}{l}
0 \\
0 \\
1
\end{array}\right)
$$

where $\lambda=c \varepsilon^{2}$ and $c$ is a constant to be fixed later.

c) If $\varepsilon \leqq r \leqq 2 \varepsilon$ we set (in the basis $(i, j, k)$ )

$$
u^{\varepsilon}(x, y)=\left(\begin{array}{c}
A_{1} r+B_{1} \\
A_{2} r+B_{2} \\
\sqrt{1-\left(A_{1} r+B_{1}\right)^{2}-\left(A_{2} r+B_{2}\right)^{2}}
\end{array}\right),
$$

where $A_{1}, A_{2}, B_{1}, B_{2}$ depend only on $\theta, \varepsilon$ are determined in such a way as to make $u^{\varepsilon}$ continuous on $\Omega$; more precisely

$$
\left\{\begin{array}{l}
2 \varepsilon A_{i}+B_{i}=\underline{u}^{i}\left(x_{0}+2 \varepsilon \cos \theta, y_{0}+2 \varepsilon \sin \theta\right) \quad i=1,2 \\
\varepsilon A_{1}+B_{1}=\frac{2 \lambda \varepsilon}{\lambda^{2}+\varepsilon^{2}} \cos \theta \\
\varepsilon A_{2}+B_{2}=\frac{2 \lambda \varepsilon}{\lambda^{2}+\varepsilon^{2}} \sin \theta
\end{array}\right.
$$

Clearly we have, as $\varepsilon \rightarrow 0$,

$$
\int_{[r>2 \varepsilon]}\left|\nabla u^{\varepsilon}\right|^{2}=\int_{[r>2 \varepsilon]}|\nabla \underline{u}|^{2}=\int_{\Omega}|\nabla \underline{u}|^{2}-4 \pi\left(a^{2}+b^{2}\right) \varepsilon^{2}+o\left(\varepsilon^{2}\right) .
$$

We claim that

$\int_{[\varepsilon<r<2 \varepsilon]}\left|\nabla u^{\varepsilon}\right|^{2}=4 \pi \varepsilon^{2}\left[a^{2}+b^{2}-2 c^{2}+\left(a^{2}+b^{2}+8 c^{2}-4 a c-4 b c\right) \log 2\right]+o\left(\varepsilon^{2}\right)$,

and

$$
\int_{[r<\varepsilon]}\left|\nabla u^{\varepsilon}\right|^{2}=8 \pi-8 \pi \varepsilon^{2} c^{2}+o\left(\varepsilon^{2}\right)
$$

We postpone for a moment the verification of (5) and (6). Combining (4), (5), and (6) we find

$$
\int_{\Omega}\left|\nabla u^{\varepsilon}\right|^{2}=\int_{\Omega}|\nabla u|^{2}+8 \pi-4 \pi \varepsilon^{2}\left[4 c^{2}-\left(8 c^{2}+a^{2}+b^{2}-4 a c-4 b c\right) \log 2\right]+o\left(\varepsilon^{2}\right) .
$$

We choose $c$ in such a way that

$$
4 c^{2}-\left(8 c^{2}+a^{2}+b^{2}-4 a c-4 b c\right) \log 2>0
$$

1 A related construction appears in [12] 
for example $c=\operatorname{Max}\{a / 2, b / 2\})$. Therefore $v=u^{\varepsilon}$ satisfies (3) provided $\varepsilon$ is small enough.

Verification of (5). We have

$$
\begin{aligned}
& A_{1}=2(a-c) \cos \theta+o(1), \\
& B_{1}=2 \varepsilon(2 c-a) \cos \theta+o(\varepsilon), \\
& A_{2}=2(b-c) \sin \theta+o(1), \\
& B_{2}=2 \varepsilon(2 c-b) \sin \theta+o(\varepsilon),
\end{aligned}
$$

and similar expressions for the $\theta$ derivatives. Thus we obtain (5) since

$$
\int_{[\varepsilon<r<2 \varepsilon]}\left|\nabla u^{\varepsilon}\right|^{2}=\int_{[\varepsilon<r<2 \varepsilon]} \sum_{i=1}^{2}\left[\left|A_{i}\right|^{2}+\left(A_{i \theta}+\frac{B_{i \theta}}{r}\right)^{2}\right] r d r d \theta .
$$

Verification of (6). We have $\left|\nabla u^{\varepsilon}\right|^{2}=8 \lambda^{2} /\left(\lambda^{2}+r^{2}\right)^{2}$ and therefore

$$
\int_{[r<\varepsilon]}\left|\nabla u^{\varepsilon}\right|^{2}=16 \pi \lambda^{2} \int_{0}^{\varepsilon} \frac{r d r}{\left(\lambda^{2}+r^{2}\right)^{2}}
$$

which leads to (6).

We turn now to property (2). We claim that

and

$$
Q\left(u^{\varepsilon}\right)=Q(\underline{u})-1+0\left(\varepsilon^{2}\right) . \quad \text { if } \quad \mathbf{i} \cdot \mathbf{j} \wedge \mathbf{k}=+1,
$$

$$
Q\left(u^{\varepsilon}\right)=Q(\underline{u})+1+0\left(\varepsilon^{2}\right) \quad \text { if } \quad \mathbf{i} \cdot \mathbf{j} \wedge \mathbf{k}=-1 .
$$

We shall verify only (7) (the proof of $\left(7^{\prime}\right)$ is identical). We write

$$
\begin{aligned}
Q\left(u^{\varepsilon}\right) & =\frac{1}{4 \pi} \int_{\Omega} u^{\varepsilon} \cdot u_{x}^{\varepsilon} \wedge u_{y}^{\varepsilon}=\frac{1}{4 \pi} \int_{[r>2 \varepsilon]}+\frac{1}{4 \pi} \int_{[\varepsilon<r<2 \varepsilon]}+\frac{1}{4 \pi} \int_{[r<\varepsilon]} \\
& \equiv I+I I+I I I .
\end{aligned}
$$

We have

and

$$
I=Q(\underline{u})+0\left(\varepsilon^{2}\right)
$$

$$
|I I| \leqq \frac{1}{8 \pi} \int_{[\varepsilon<r<2 \varepsilon]}\left|\nabla u^{\varepsilon}\right|^{2}=0\left(\varepsilon^{2}\right) \text { by (5) }
$$

In order to evaluate $I I I$ we note that in the region $[r<\varepsilon]$ we have

$$
u^{\varepsilon} \cdot u_{x}^{\varepsilon} \wedge u_{y}^{\varepsilon}=-\frac{8 \lambda^{4}}{\left(\lambda^{2}+r^{2}\right)^{3}}+\mathbf{k} \cdot u_{x}^{\varepsilon} \wedge u_{y}^{\varepsilon}
$$

and thus by (5),

$$
I I I=-4 \int_{0}^{\varepsilon} \frac{\lambda^{4} r d r}{\left(\lambda^{2}+r^{2}\right)^{3}}+0\left(\varepsilon^{2}\right)=-1+0\left(\varepsilon^{2}\right) .
$$

Combining (8), (9), and (10) we obtain (7). 
Remark. 1. The conclusion of Lemma 2 asserts that there is some $v \in \mathscr{E}$ such that $Q(v)-Q(\underline{u})= \pm 1$, and $E(v)<E(\underline{u})+8 \pi$.

In general one can not find two $v$ 's $v_{1}, v_{2} \in \mathscr{E}$ such that

$$
\left\{\begin{array}{l}
Q\left(v_{1}\right)-Q(\underline{u})=+1 \\
Q\left(v_{2}\right)-Q(\underline{u})=-1 \\
E\left(v_{i}\right)<E(\underline{u})+8 \pi, \quad i=1,2 .
\end{array}\right.
$$

When (11) happens to be true one can prove that both $\operatorname{Inf}_{\mathscr{E}+1} E$ and $\operatorname{Inf}_{\mathscr{E}} E$ are achieved (see the proof of Theorem 1). [However there are simple examples where only one of these two infima is achieved (see Sect. 3).] Notice that (11) holds in the following cases:

a) There is some point $\left(x_{0}, y_{0}\right) \in \Omega$ such that

$$
\begin{gathered}
\nabla \underline{u} \neq 0 \text { at }\left(x_{0}, y_{0}\right), \\
\underline{u} \cdot \underline{u}_{x} \wedge \underline{u}_{y}=0 \text { at }\left(x_{0}, y_{0}\right) .
\end{gathered}
$$

b) There are two points $\left(x_{0}, y_{0}\right) \in \Omega,\left(x_{1}, y_{1}\right) \in \Omega$ such that

$$
\begin{array}{lll}
\underline{u} \cdot \underline{u}_{x} \wedge \underline{u}_{y}>0 & \text { at } & \left(x_{0}, y_{0}\right), \\
\underline{u} \cdot \underline{u}_{x} \wedge \underline{u}_{y}<0 & \text { at } & \left(x_{1}, y_{1}\right) .
\end{array}
$$

[This is a direct consequence of the argument we have used in the proof of Lemma 2.]

\section{Proof of Theorem 1.}

Let $v \in \mathscr{E}$ be given by Lemma 2. We shall establish that if $v \in \mathscr{E}_{1}$ (respectively $v \in \mathscr{E}_{-1}$ ) then $\operatorname{Inf} E($ respectively $\operatorname{Inf} E)$ is achieved. We consider just the case where $v \in \mathscr{E}_{1}$ (the other case is similar). Let $\left(u^{n}\right)$ be a minimizing sequence, i.e. $u^{n} \in \mathscr{E}_{1}$ and $E\left(u^{n}\right)=\operatorname{Inf}_{\mathscr{E}_{1}} E$ $+o(1)($ as $n \rightarrow \infty)$. We may extract a subsequence still denoted by $u^{n}$ such that $u^{n} \rightarrow \bar{u}$ weakly in $H^{1}\left(\Omega ; \mathbb{R}^{3}\right)$. Clearly $\bar{u} \in \mathscr{E}$ and $E(\bar{u}) \leqq \operatorname{Inf}_{\mathscr{E}_{1}} E$. It remains to prove that $\bar{u} \in \mathscr{E}_{1}$. Suppose by contradiction that $\bar{u} \notin \mathscr{E}_{1}$. It follows that

$$
\left|Q\left(u^{n}\right)-Q(\bar{u})\right| \geqq 1 \text {. }
$$

Assume for example that

$$
Q\left(u^{n}\right) \geqq Q(\bar{u})+1
$$

Set

$$
F(v)=E(v)-8 \pi Q(v)=\int_{\Omega}|\nabla v|^{2}-2 \int_{\Omega} v \cdot\left(v_{x} \wedge v_{y}\right)
$$

Using the same argument as in [2] (see the proof of Lemma 1) one obtains

$$
F(\bar{u}) \leqq \lim \inf F\left(u^{n}\right) \text {. }
$$

Combining (13), (14) and (15) we find

$$
E(\bar{u})-8 \pi Q(\bar{u}) \leqq \lim \inf \left\{E\left(u^{n}\right)-8 \pi Q(\bar{u})-8 \pi\right\} .
$$


Hence

$$
E(\bar{u}) \leqq \operatorname{Inf}_{\mathscr{E}_{1}} E-8 \pi .
$$

On the other hand, by Lemma 2, there is some $v \in \mathscr{E}_{1}$ such that

$$
E(v)<E(\underline{u})+8 \pi,
$$

a contradiction with (16).

Remark. 2. The conclusion of Theorem 1 still holds if we replace $S^{2}$ by a Riemannian surface $M$ homeomorphic to $S^{2}$. Using a conformal diffeomorphism between $M$ and $S^{2}$, this amounts to establish Theorem 1 for $E^{\prime}(u)=\int_{\Omega} g(u)|\nabla u|^{2}$ instead of $E$, where $g \in C^{1}\left(S^{2} ;(0, \infty)\right)$ and $u: \Omega \rightarrow S^{2}$. We replace $Q$ by

$$
Q^{\prime}(u)=\frac{1}{\Sigma} \int_{\Omega} g(u) u \cdot u_{x} \wedge u_{y}
$$

where $\Sigma=\int_{S^{2}} g d \sigma$ and $u: \Omega \rightarrow S^{2}$. Instead of Lemma 1 and 2 we have now, with nearly the same proofs:

Lemma 1'. Assume $u_{1}, u_{2} \in \mathscr{E}$, then $Q^{\prime}\left(u_{1}\right)-Q^{\prime}\left(u_{2}\right) \in \mathbb{Z}$.

Let $u^{\prime} \in \mathscr{E}$ be such that $E^{\prime}\left(u^{\prime}\right)=\operatorname{Inf}_{\mathscr{E}} E^{\prime}$.

Lemma 2'. There is some $v \in \mathscr{E}$ such that $\left|Q^{\prime}(v)-Q^{\prime}\left(u^{\prime}\right)\right|=1$ and $E^{\prime}(v)<E^{\prime}\left(u^{\prime}\right)+2^{\varepsilon}$. Then we proceed as in the proof of Theorem 1 .

\section{A Simple Example}

We consider now the case where $\gamma(x, y)=\left(R x, R y, \sqrt{1-R^{2}}\right)$ for $(x, y) \in \partial \Omega$ with $0<R<1$. In that case we shall give a complete description of the solution of the problems $\operatorname{Inf} E$ and $\operatorname{Inf} E$. For this purpose, we set

$$
\begin{aligned}
& \lambda=\frac{1}{R}+\sqrt{\frac{1}{R^{2}}-1}, \quad \mu=\frac{1}{R}-\sqrt{\frac{1}{R^{2}}-1} \\
& \underline{u}(x, y)=\frac{2 \lambda}{\lambda^{2}+r^{2}}\left(\begin{array}{l}
x \\
y \\
\lambda
\end{array}\right)+\left(\begin{array}{r}
0 \\
0 \\
-1
\end{array}\right), \quad \bar{u}(x, y)=\frac{2 \mu}{\mu^{2}+r^{2}}\left(\begin{array}{r}
x \\
y \\
-\mu
\end{array}\right)+\left(\begin{array}{l}
0 \\
0 \\
1
\end{array}\right),
\end{aligned}
$$

with $(x, y) \in \Omega$ and $r^{2}=x^{2}+y^{2}$.

Theorem 2. We have

(A) $\underline{u} \in \mathscr{E}$ and $E(\underline{u})=\operatorname{Inf}_{\mathscr{E}} E$;

moreover $\underline{u}$ is the unique element which minimizes $E$ on $\mathscr{E}$.

(B) $\bar{u} \in \mathscr{E}_{-1}$ and $E(\bar{u})=\operatorname{Inf}_{\mathscr{E}-1} E$;

moreover $\bar{u}$ is the unique element which minimizes $E$ on $\mathscr{E}_{-1}$.

(C) $\operatorname{Inf} E$ is not achieved if $k \notin\{0,-1\}$. 
Proof. Part $A$. Let $\tilde{u} \in \mathscr{E}$ be such that

First we claim that

$$
E(\tilde{u}) \leqq E(v) \quad \forall v \in \mathscr{E} .
$$

$$
Q(\tilde{u})=Q(\underline{u}) \text {. }
$$

Assume by contradiction that $|Q(\tilde{u})-Q(\underline{u})| \geqq 1$ (see Lemma 1). We introduce $w: \mathbb{R}^{2} \rightarrow S^{2}$ defined as follows:

$$
\left\{\begin{array}{l}
w(x y)=\tilde{u}(x, y) \text { for } r<1 \\
w(x, y)=\underline{u}\left(\frac{x}{r^{2}}, \frac{y}{r^{2}}\right) \text { for } r>1,
\end{array}\right.
$$

so that $w \in L^{\infty}\left(\mathbb{R}^{2}, S^{2}\right)$ and $w_{x}, w_{y} \in L^{2}\left(\mathbb{R}^{2} ; \mathbb{R}^{3}\right)$. By the proof of Lemma 1 we have

$$
\frac{1}{4 \pi} \int_{\mathbb{R}^{2}} w \cdot w_{x} \wedge w_{y}=Q(\tilde{u})-Q(\underline{u}),
$$

and thus

$$
\frac{1}{4 \pi}\left|\int_{\mathbb{R}^{2}} w \cdot w_{x} \wedge w_{y}\right| \geqq 1
$$

Therefore we obtain

$$
\int_{\mathbb{R}^{2}}|\nabla w|^{2} \geqq 8 \pi
$$

Obviously we have

$$
\int_{\mathbb{R}^{2}}|\nabla w|^{2}=E(\tilde{u})^{\prime}+E(\underline{u})
$$

and a direct computation shows that

$$
E(\underline{u})=4 \pi\left(1-\sqrt{1-R^{2}}\right) .
$$

Combining (19), (20) and (21) we obtain

$$
E(\tilde{u}) \geqq 4 \pi\left(1+\sqrt{1-R^{2}}\right)>E(\underline{u})
$$

- a contradiction with (17). Hence we have proved (18).

Next we consider the function $\bar{w}: \mathbb{R}^{2} \rightarrow S^{2}$ defined as follows:

$$
\left\{\begin{array}{l}
\bar{w}(x, y)=\tilde{u}(x, y) \text { for } r<1 \\
\bar{w}(x, y)=\bar{u}\left(\frac{x}{r^{2}}, \frac{y}{r^{2}}\right) \text { for } r>1
\end{array}\right.
$$

We have

$$
\int_{\mathbb{R}^{2}}|\nabla \bar{w}|^{2}=E(\tilde{u})^{\prime}+E(\bar{u}) \leqq E(\underline{u})^{\prime}+E(\bar{u}),
$$

and a direct computation shows that

$$
E(\bar{u})=4 \pi\left(1+\sqrt{1-R^{2}}\right) .
$$


Combining (21), (22), and (23) we see that

$$
\int_{\mathbb{R}^{2}}|\nabla \bar{w}|^{2} \leqq 8 \pi
$$

Moreover, we have (using (18)),

$$
\frac{1}{4 \pi} \int_{\mathbb{R}^{2}} \bar{w} \cdot \bar{w}_{x} \wedge \bar{w}_{y}=Q(\tilde{u})-Q(\bar{u})=Q(\underline{u})-Q(\bar{u})=1
$$

(the last equality follows from a direct computation). Thus, $\int_{\mathbb{R}^{2}}|\nabla \bar{w}|^{2} \geqq 8 \pi$ and in fact (by (24)), $\int_{\mathbb{R}^{2}}|\nabla \bar{w}|^{2}=8 \pi$. The conclusion of Lemma A.2 asserts that $\bar{w}$ is analytic on $\mathbb{R}^{2}$. Finally we consider $\overline{\bar{w}}: \mathbb{R}^{2} \rightarrow S^{2}$ defined as follows:

$$
\left\{\begin{array}{l}
\overline{\bar{w}}(x, y)=\underline{u}(x, y) \text { for } r<1 \\
\overline{\bar{w}}(x, y)=\bar{u}\left(\frac{x}{r^{2}}, \frac{y}{r^{2}}\right) \text { for } r>1 .
\end{array}\right.
$$

It is readily seen (by direct inspection) that $\bar{w}$ is analytic in $\mathbb{R}^{2}$. On the other hand we have $\overline{\bar{w}}=\bar{w}$ for $r>1$, and therefore $\overline{\bar{w}}=\bar{w}$, i.e. $\underline{u}=\tilde{u}$.

Part $B$. Let $v \in \mathscr{E}_{-1}$; we shall first check that

$$
E(\bar{u}) \leqq E(v) .
$$

Let $w: \mathbb{R}^{2} \rightarrow S^{2}$ be defined as follows:

We have

$$
\left\{\begin{array}{l}
w(x, y)=v(x, y) \text { for } r<1 \\
w(x, y)=\underline{u}\left(\frac{x}{r^{2}}, \frac{y}{r^{2}}\right) \text { for } r>1 .
\end{array}\right.
$$

$$
\int_{\mathbb{R}^{2}}|\nabla w|^{2}=E(v)+E(\underline{u})
$$

and moreover

$$
\frac{1}{4 \pi} \int_{\mathbb{R}^{2}} w \cdot w_{x} \wedge w_{y}=Q(v)-Q(\underline{u})=-1
$$

Thus

$$
\int|\nabla w|^{2} \geqq 8 \pi \text {. }
$$

Combining (26), (28), (21), and (23) we obtain (25).

Finally we assume in addition that

$$
E(v)=E(\bar{u}) \quad \text { with } v \in \mathscr{E}_{-1} .
$$

We deduce from (26), (29), (21), and (23) that

$$
\int_{\mathbb{R}^{2}}|\nabla w|^{2}=8 \pi
$$

Again, by Lemma A.2, $w$ is analytic on $\mathbb{R}^{2}$ and we conclude as in Part A that $v=\bar{u}$. 
Part $C$. We assume for example that $k>0$ (the argument is similar for $k \leqq-2$ ). Suppose, by contradiction, that there is some $v \in \mathscr{E}_{k}$ such that

$$
E(v)=\operatorname{Inf}_{\mathscr{E}_{k}} E .
$$

It is a well known fact that

$$
\operatorname{Inf}_{\mathscr{E}_{k}} E \leqq E(\underline{u})+8 k \pi .
$$

[The technique is similar to the one used in the proof of Lemma 2, except that it is much simpler since we don't require a strict inequality. Given $\varepsilon>0$, one considers, for example, $v^{\varepsilon}: \Omega \rightarrow S^{2}$ such that

a) If $r>2 \varepsilon, v^{\varepsilon}(x, y)=\underline{u}(x, y)$.

b) If $r<\varepsilon$ we set

$$
v^{\varepsilon}(x, y)=\frac{2 \varepsilon^{k+1}}{\varepsilon^{2 k+2}+r^{2 k}}\left(\begin{array}{c}
r^{k} \cos k \theta \\
-r^{k} \sin k \theta \\
-\varepsilon^{k+1}
\end{array}\right)+\left(\begin{array}{l}
0 \\
0 \\
1
\end{array}\right)
$$

c) If $\varepsilon<r<2 \varepsilon$ we proceed as in the proof of Lemma 2 .

One checks that $v^{\varepsilon} \in \mathscr{E}_{k}$ and $\left.E\left(v^{\varepsilon}\right)=E(\underline{u})+8 k \pi+o(1) \cdot\right]$

Finally we consider the function $w: \mathbb{R}^{2} \rightarrow S^{2}$ defined as follows:

so that

$$
\begin{cases}w(x, y)=v(x, y) & \text { for } r<1 \\ w(x, y)=\bar{u}\left(\frac{x}{r^{2}}, \frac{y}{r^{2}}\right) & \text { for } r>1\end{cases}
$$

$$
\frac{1}{4 \pi} \int_{\mathbb{R}^{2}} w \cdot w_{x} \wedge w_{y}=Q(v)-Q(\bar{u})=k+1 .
$$

We deduce from (30) and (31) that

$$
\int_{\mathbb{R}^{2}}|\nabla w|^{2}=E(v)+E(\bar{u}) \leqq E(\underline{u})+E(\bar{u})+8 k \pi=8(k+1) \pi .
$$

Once more it follows from Lemma A.2 that $w$ is analytic and thus (as in Part $A$ ), $v=\underline{u}$-a contradiction since $v \in \mathscr{E}_{k}(k \neq 0)$.

\section{Appendix}

We start with a useful density result due to Schoen-Uhlenbeck [10]. For the convenience of the reader we sketch its proof.

Lemma A.1. Given $u \in L^{\infty}\left(\mathbb{R}^{2} ; S^{2}\right)$ with $\nabla u \in L^{2}\left(\mathbb{R}^{2} ; \mathbb{R}^{6}\right)$ there exists a sequence $\left(u_{n}\right)$ such that

$$
\left\{\begin{array}{l}
u_{n} \in C^{\infty}\left(\mathbb{R}^{2} ; S^{2}\right) \\
\text { each } u_{n} \text { is constant far out, } \\
u_{n} \rightarrow u \text { a.e. } \\
\nabla u_{n} \rightarrow \nabla u \text { in } L^{2}\left(\mathbb{R}^{2} ; \mathbb{R}^{6}\right)
\end{array}\right.
$$


Proof. We denote by $\pi: S^{2} \rightarrow \mathbb{R}^{2}$ the stereographic projection which maps the south pole into 0 . We set $v(p)=u(\pi(p))$ for $p \in S^{2}$. It is well known that $v \in H^{1}\left(S^{2} ; S^{2}\right)$. Let $v_{n}(p)$ denote the average of $v$ over $B_{1 / n}(p)=\left\{q \in S^{2} ;|q-p|<1 / n\right\}$ and thus we have

$$
v_{n} \in C\left(S^{2} ; \mathbb{R}^{2}\right) \cap H^{1}\left(S^{2} ; \mathbb{R}^{2}\right),
$$

and $v_{n} \rightarrow v$ in $H^{1}\left(S^{2} ; \mathbb{R}^{3}\right)$. Note that $v_{n}$ does not take its values into $S^{2}$. However Poincaré's inequality shows that

$$
\int_{B_{1 / n}(p)}\left|v(q)-v_{n}(p)\right| d q \leqq \frac{C}{n^{2}}\left(\int_{B_{1 / n}(p)}|\nabla v|^{2}\right)^{1 / 2}
$$

and therefore

$$
\operatorname{dist}\left(v_{n}(p), S^{2}\right) \underset{n \rightarrow \infty}{\rightarrow} 0 \text { uniformly in } p \in S^{2} .
$$

By a small modification of $v_{n}$ we may as well assume that

$$
\left\{\begin{array}{l}
v_{n} \in C^{\infty}\left(S^{2} ; \mathbb{R}^{2}\right), \\
\text { each } v_{n} \text { is constant near the north pole, } \\
v_{n} \rightarrow v \text { in } H^{1}\left(S^{2} ; \mathbb{R}^{3}\right), \\
\operatorname{dist}\left(v_{n}(p), S^{2}\right) \rightarrow 0 \text { uniformly in } p \in S^{2}
\end{array}\right.
$$

Projecting $v_{n}(p)$ on $S^{2}$ we may further assume that $v_{n}(p) \in S^{2} \forall n, \forall p \in S^{2}$. The sequence $u_{n}(x, y)=v_{n}\left(\pi^{-1}(x, y)\right)$ satisfies all the required properties.

In our next lemma we extend to Sobolev classes a property which is well known for smooth maps.

\section{Lemma A.2. We have}

$$
\int_{\mathbb{R}^{2}}|\nabla \phi|^{2} \geqq 2\left|\int_{\mathbb{R}^{2}} \phi \cdot \phi_{x} \wedge \phi_{y}\right| \forall \phi \in L^{\infty}\left(\mathbb{R}^{2} ; S^{2}\right) \quad \text { with } \phi_{x}, \phi_{y} \in L^{2}\left(\mathbb{R}^{2} ; \mathbb{R}^{3}\right)
$$

and if equality holds in $(*)$, then $\phi$ is analytic.

Proof. Inequality $\left(^{*}\right)$ is trivial since $|\phi|=1$. Suppose now that we have some $\phi \in L^{\infty}\left(\mathbb{R}^{2} ; S^{2}\right)$ with $\phi_{x}, \phi_{y} \in L^{2}\left(\mathbb{R}^{2} ; \mathbb{R}^{3}\right)$, and such that

$$
\int_{\mathbb{R}^{2}}|\nabla \phi|^{2}=2 \int_{\mathbb{R}^{2}} \phi \cdot \phi_{x} \wedge \phi_{y}
$$

We shall prove that $\phi \in C^{\infty}\left(\mathbb{R}^{2} ; S^{2}\right)$. This will imply that $\phi$ is analytic. Indeed if equality in (A.1) holds then $\phi$ is a harmonic map and thus $\phi$ is analytic.

We now prove that $\phi$ is $C^{\infty}$ for example near 0 . We fix $\rho>0$ such that

$$
\int_{D}|\nabla \phi|^{2} \leqq 2 \pi
$$

where $D=\left\{(x, y) \in \mathbb{R}^{2} ; x^{2}+y^{2}<\rho^{2}\right\}$ and we let $\gamma=\left.\phi\right|_{\partial D}$. We claim that

$$
\int_{D}|\nabla \phi|^{2} \leqq \int_{D}|\nabla \psi|^{2}, \forall \psi \in H^{1}\left(D ; S^{2}\right), \psi=\gamma \quad \text { on } \partial D
$$


-which in turn implies that $\phi \in C^{\infty}\left(D ; S^{2}\right)$ by Morrey's regularity theory.

In order to establish (A.3) we assume by contradiction that there is some $\psi \in H^{1}\left(D ; S^{2}\right)$ with $\psi=\gamma$ on $\partial D$ and

$$
\int_{D}|\nabla \psi|^{2}<\int_{D}|\nabla \phi|^{2}
$$

We have

$$
\int_{D} \psi \cdot \psi_{x} \wedge \psi_{y} \neq \int_{D} \phi \cdot \phi_{x} \wedge \phi_{y}
$$

Indeed if we had

$$
\int_{D} \psi \cdot \psi_{x} \wedge \psi_{y}=\int_{D} \phi \cdot \phi_{x} \wedge \phi_{y}
$$

we could introduce the map $\tilde{\phi}: \mathbb{R}^{2} \rightarrow S^{2}$ defined as follows:

$$
\begin{cases}\tilde{\phi}=\psi & \text { on } D \\ \tilde{\phi}=\phi & \text { on } \mathbb{R}^{2} \backslash \mathrm{D},\end{cases}
$$

and we would find

$$
\int_{\mathbb{R}^{2}} \tilde{\phi} \cdot \tilde{\phi}_{x} \wedge \tilde{\phi}_{y}=\int_{\mathbb{R}^{2}} \phi \cdot \phi_{x} \wedge \phi_{y}
$$

and

$$
\int_{\mathbb{R}^{2}}|\nabla \phi|^{2}=\int_{D}|\nabla \psi|^{2}+\int_{\mathbb{R}^{2} \backslash D}|\nabla \phi|^{2}<\int_{\mathbb{R}^{2}}|\nabla \phi|^{2} \quad \text { (by (A.4)). }
$$

Applying (*) to $\delta$ and combining the resulting inequality with (A.1) and (A.6) we would obtain a contradiction. Thus we have established (A.5).

Finally we consider the map $h: \mathbb{R}^{2} \rightarrow S^{2}$ defined as follows:

$$
\left\{\begin{array}{l}
h=\psi \text { in } D \\
h(x, y)=\phi\left(\frac{\rho^{2} x}{x^{2}+y^{2}}, \frac{\rho^{2} y}{x^{2}+y^{2}}\right) \text { for }(x, y) \in \mathbb{R}^{2} \backslash D
\end{array}\right.
$$

so that $h \in L^{\infty}\left(\mathbb{R}^{2} ; S^{2}\right), h_{x}, h_{y} \in L^{2}\left(\mathbb{R}^{2} ; \mathbb{R}^{3}\right)$ and

$$
\int_{\mathbb{R}^{2}} h \cdot h_{x} \wedge h_{y}=\int_{D} \psi \cdot \psi_{x} \wedge \psi_{y}-\int_{D} \phi \cdot \phi_{x} \wedge \phi_{y} .
$$

We deduce from (A.5) that

$$
\left|\int_{\mathbb{R}^{2}} h \cdot h_{x} \wedge h_{y}\right| \geqq 4 \pi,
$$

and thus $\int_{\mathbb{R}^{2}}|\nabla h|^{2} \geqq 8 \pi$. But

$$
\int_{\mathbb{R}^{2}}|\nabla h|^{2}=\int_{D}|\nabla \psi|^{2}+\int_{D}|\nabla \phi|^{2} \leqq 2 \int_{D}|\nabla \phi|^{2}
$$

(by (A.4)). Hence $\int_{D}|\nabla \phi|^{2} \geqq 4 \pi$ - a contradiction with (A.2). 
Acknowledgements. We thank S. Hildebrandt for drawing our attention to this problem which is raised in [4]. This paper was written while both authors were visiting Princeton University. We thank E. Lieb, the Mathematics Department and the Physics Department for their invitation and kind hospitality.

\section{References}

1. Aubin, Th.: Equations différentielles non linéaires et problème de Yamabe concernant la courbure scalaire. J. Math. Pures Appl. 55, 269-296 (1976)

2. Brezis, H., Coron, J. M.: Multiple solutions of H-systems and Rellich's conjecture. Commun. Pure Appl. Math. (to appear)

3. Brezis, H. Nirenberg, L.: Positive solutions of nonlinear elliptic equations involving critical Sobolev exponents. Commun. Pure Appl. Math. (to appear)

4. Giaquinta, M., Hildebrandt, S.: A priori estimates for harmonic mappings. J. Reine Angew. Math. 336, 124-164 (1982)

5. Jost, J.: The Dirichlet problem for harmonic maps from a surface with boundary onto a 2-sphere with nonconstant boundary values. Invent. Math. (to appear)

6. Lemaire, L.: Applications harmoniques de surfaces riemanniennes. J. Diff. Geom. 13, 51-78 (1978)

7. Lieb, E.:Sharp constants in the Hardy-Littlewood-Sobolev and related inequalities. Ann. Math. (to appear)

8. Lions, P. L.: The concentration-compactness principle in the Calculus of Variations; The limit case. (to appear)

9. Nirenberg, L.: Topics in nonlinear functional analysis, New York University Lecture Notes 19731974

10. Schoen, R., Uhlenbeck, K.: Boundary regularity and miscellaneous results on harmonic maps. J. Diff. Geom. (to appear)

11. Taubes, C.: The existence of a non-minimal solution to the SU(2) Yang-Mills-Higgs equations on $\mathbb{R}^{3}$. Commun. Math. Phys. 86, 257-298, 299-320 (1982)

12. Wente, H.: The Dirichlet problem with a volume constraint, Manuscripta Math. 11, 141-157 (1974)

Communicated by A. Jaffe

Received April 11, 1983, in revised form June 20, 1983 
